selected for outcome analyses. Among seropositive patients, both LDA and remission were less frequently achieved in case of RF-positivity. Relevantly, in ACPA-positive patients $(n=273)$, the co-occurrence of RF dose-dependently influenced clinical outcomes. After 6 months of treatment, LDA and disease remission were achieved respectively by $69.6 \%$ and $47.8 \%$ of single ACPApositive, $68.8 \%$ and $37.5 \%$ of ACPA-positive RF-low, and $57 \%$ and $27.6 \%$ of ACPA-positive RF-high patients (chi-square for trend $p=0.18$ for LDA, $p=0.05$ for remission). After adjusting for confounders (age, gender, symptoms' duration, baseline disease activity, use of prednisone, recruitment period), high levels of RF independently predicted failure to achieve LDA with an $\mathrm{HR}(95 \% \mathrm{Cl})$ of 0.61 (0.39 to 0.95$)$ and failure to achieve remission with an $\mathrm{HR}(95 \% \mathrm{Cl})$ of $0.63(0.35$ to 0.99 ) (Figure 1). In contrast, ACPA levels did not show any significant predictive value, neither for thresholds of $>3 \mathrm{ULN}$ nor of $>100 \mathrm{U}$.

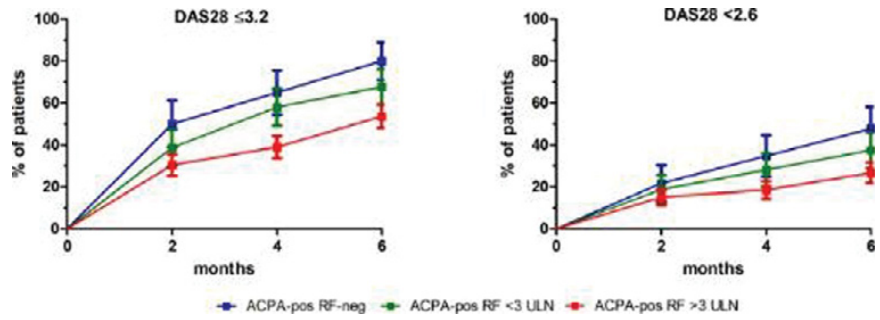

Conclusions: Among ACPA-positive RA patients, disease characteristics may vary in association with the extent of overall humoral autoimmunity. In particular, the concomitant presence of high levels of IgM RF seems associated with lower response rates to csDMARDs. Collectively, these findings highlight the importance of further subclassifying patients with autoantibody-positive RA in order get deeper insights into disease mechanisms and clinical outcomes. References:

[1] Aletaha D et al. Arthritis Res Ther 2015;17:229.

[2] Derksen VFAM et al. Ann Rheum Dis 2017; doi:10.1136/annrheumdis-2016209794.

[3] Sokolove J et al. Arthritis Rheumatol 2014;66:813-21.

Disclosure of Interest: None declared

DOI: 10.1136/annrheumdis-2017-eular.5778

\section{SAT0061 HAQ SCORE IS AN INDEPENDENT PREDICTOR OF SUSTAINED REMISSION IN PATIENTS WITH RHEUMATOID ARTHRITIS}

S.-S. Lee ${ }^{1,1}$, J.-H. Kang, J.-E. Kim, K.-E. Lee, D.-J. Park. Chonnam National University Medical School and Hospital, Gwangju, Korea, Republic Of

Objectives: We compared remission rates, according to different definitions of remission in rheumatoid arthritis (RA) and investigated the potential predictors of sustained remission at the 2-year follow-up.

Methods: We obtained data on 291 RA outpatients, seen from July 2009 to September 2012. Sociodemographic data and answers to questionnaires were collected in face-to-face interviews. Remission was defined according to the Disease Activity Score in 28 joints (DAS28)-ESR, DAS28-CRP, Simplified Disease Activity Index (SDAI), Clinical Disease Activity Index (CDAl), and ACR/EULAR Boolean definition. Sustained remission was defined as when the patient continued in remission at two consecutive annual assessments. Predictors of sustained remission according to the DAS28-CRP were assessed by univariate and multivariate analyses.

Results: For the 291 RA patients, the remission rates of RA were $17.9 \%$ (DAS28-ESR), 54.3\% (DAS28-CRP), 10.3\% (SDAI), $10.0 \%$ (CDAI), and $5.8 \%$ (Boolean). On follow-up for 2 years, the sustained remission rates of RA were 46.5\% (DAS28), $55.0 \%$ (DAS28-CRP), 37.5\% (SDAl), 32.0\% (CDAl), and $30.8 \%$ (Boolean). RA patients who achieve sustained remission according to the DAS28-CRP were younger, and had more education, higher monthly income, lower Health Assessment Questionnaire (HAQ) score, lower physician global assessment, lower patient global assessment, lower patient pain assessment, and higher EQ-5D. In multivariate analysis, only the HAQ score predicted sustained remission according to DAS28-CRP (OR=0.257, 95\% Cl 0.067-0.980, $p=0.047$ ). Conclusions: The remission rates of RA patients differed according to the definition of remission, and the highest sustained remission rate was classified by the DAS28-CRP. A lower HAQ score was an independent predictor of sustained remission over 2 years, according to the DAS28-CRP.

Disclosure of Interest: None declared

DOI: 10.1136/annrheumdis-2017-eular.2363

\section{SAT0062 14-3-3ETA PREDICTS JOINT DAMAGE PROGRESSION AND FLARING AFTER ADALIMUMAB DISCONTINUATION}

S. Hirata ${ }^{1,2}$, A. Marotta ${ }^{3}$, K. Hanami ${ }^{2}$, Y. Tanaka ${ }^{2} .{ }^{1}$ Hiroshima University Hospital, Hiroshima; ${ }^{2}$ University of Occupational and Environmental Health, Kitakyushu, Japan; ${ }^{3}$ Augurex Life Sciences Corp, Vancouver, Canada

Background: The HONOR (humira discontinuation without functional and radio- graphic damage following sustained remission) study was designed to investigate the possibility of patients discontinuing adalimumab (ADA) therapy for 1 year without flaring (DAS28-ESR $\geq 3.2$ ). $14-3-3 \eta$ is a mechanistic serum marker that is modifiable over the disease course, does not correlate with CRP and is a predictor of radiographic progression even in patients who achieve clinical remission. The uncoupling of inflammation and joint damage processes in RA underscores a risk of premature discontinuation of biologic treatment when aiming to achieve sustained remission. Serum markers that could indicate which patients are at risk of flare and continued joint damage despite clinical remission are highly desirable.

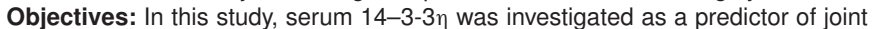
damage and flares in the HONOR cohort.

Methods: Serum 14-3-3n levels were measured in 62 Japanese patients, 51 of which were from the HONOR study at baseline, 1-year after treatment initiation, at discontinuation and at the time of flare. Of the 62 patients, $46(74 \%)$ patients achieved sustained drug-free remission up to 1 year following ADA discontinuation. Sharp van der Heidje (SHS) scores were available at therapy initiation, discontinuation, and at 52 weeks following. Relationships between continuous variables were assessed using uni- and multi-variable Gaussian linear regression models and logistic regression.

Results: At baseline and discontinuation, median (QR) $14-3-3 \eta$ levels were 0.28 $\mathrm{ng} / \mathrm{ml}(0.07-2.11)$ and $0.22 \mathrm{ng} / \mathrm{ml}(0.04-1.28)$ respectively, with $26(59 \%)$ of 44 and $29(54 \%)$ of 54 patients being positive $(\geq 0.19 \mathrm{ng} / \mathrm{ml})$ at the corresponding time-points. Paired $t$-test revealed that levels of $14-3-3 \eta$ were significantly different between baseline and discontinuation, $p=0.030$. Level of $14-3-3 \eta$ at baseline was positively associated with SHS at 12 months and at the time of flare, $p=0.038$. Bivariable modeling revealed that baseline $14-3-3 \eta$ together with the change in 14-3-3 $\eta$ had a significant interacting effect on SHS at 12 months and the time of flare, $p=0.02$. Higher baseline $14-3-3 \eta$ levels together with an increase in levels at the time of discontinuation was strongly associated with an increased SHS. Adding CRP, flare, sustained remission through 12 months, MTX dose at initiation and at ADA discontinuation did not improve predictive effects of $14-3-3 \eta$ with

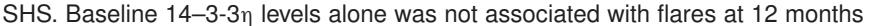
$(p=0.15)$ however when combined with CRP, a significant interaction was present $(p=0.03)$. Specifically, patients with a low CRP, and a high $14-3-3 \eta$ level had a higher likelihood of flaring versus those with a low $14-3-3 \eta, 22 \%$ versus $12 \%$.

Conclusions: Baseline 14-3-3 $\eta$ and increases in its levels are associated with worse radiographic outcomes in patients who achieve clinical remission and discontinue $A D A$. To reduce the risk of flare in patients who are candidates for

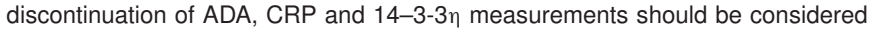
in combination as markers of flare prediction.

Disclosure of Interest: S. Hirata: None declared, A. Marotta Employee of: Augurex Life Sciences Corp., K. Hanami: None declared, Y. Tanaka: None declared DOI: 10.1136/annrheumdis-2017-eular.5426

\section{SAT0063 A PROSPECTIVE STUDY ON COMPARISON OF COMPOSITE INDICES WITH ULTRASOUND FOR DETECTING REMISSION AND PREDICTION OF FLARE IN 2 YEARS}

M.O. Olmez ${ }^{1}$, E.K. Gunal ${ }^{2}$, S.B. Ureyen ${ }^{3}$, H. Keskin ${ }^{2}$, A.B. Ozturk ${ }^{4}$, H.G. Yeter ${ }^{5}$, E. Cobanoglu ${ }^{5}$, S.Z. Aydin ${ }^{3} .{ }^{1}$ Internal Medicine; ${ }^{2}$ Rheumatology, Istanbul Medeniyet University School of Medicine, Istanbul, Turkey;

${ }^{3}$ Rheumatology, University of Ottawa, Faculty of Medicine, Ottawa, Canada; ${ }^{4}$ Allergy and Immunology; ${ }^{5}$ Koc University, School of Medicine, Istanbul, Turkey

Background: Treat-to-target (T2T) approach suggests using a composite index when following patients with rheumatoid arthritis (RA) without identifying which one to use.

Objectives: In this prospective study we aimed to compare the accuracy of different indices for RA patients in remission taking Ultrasound Global Synovitis Score (GLOESS) as a gold standard and their predictive value for flares in 2 years.

Methods: RA patients who were considered to be in clinical remission according to the clinician were recruited. Disease activity was assessed using DAS28-CRP, CDAI, SDAI and RAPID-3 and 38 joints per patient were scanned by US and scored according to GLOESS. The total GLOESS scores were calculated for 38 joints and also for 28 joints by excluding the MTP joints. The number of joints with $\geq 2$ GLOESS was calculated. Flare data was collected in 3 subsequent visits in the following 2 years, whenever available.

Results: Ninety-six consecutive patients ( $80.2 \%$ females) were recruited. Patients were more frequently categorized as being in remission using DAS28 $(80 \%)$ compared to CDAI (50\%), SDAI (45.2\%) and RAPID $3(37.5 \%)$. Patients that were in remission according to CDAI had lower GLOESS scores on 28 joints $(p=0.05)$ and had less joints with $\geq 2$ signals $(\mathrm{p}=0.04$ ) (table). For SDAI patients in remission had significantly less number of joints with grade 3 signals $(p=0.03)$ and tend to have lower GLOESS scores on 28 joints as well as lower number of joints with $\geq 2$ signals $(p=0.06)$. None of the US scores were able to differentiate different disease states according to DAS28-CRP or RAPID. Flare data was available in 76 patients, 22 of whom had flares. Patients that had flare had higher GLOESS scores on 28 joints at baseline $(p=0.05)$ and tend to have higher number of joints with grade 3 signals $(p=0.06)$. Although numerically higher, none of the clinical indices were able to predict flares based on remission status (remission vs non remission: CDAl: $22.5 \%$ vs $36.1, p=0.2$; SDAl: 22.9 vs $36.8, p=0.2$; DAS28: $25.4 \%$ vs $50 \%, p=0.1$; RAPID3: $20 \%$ vs $34.8 \%, p=0.2$ ) 